\title{
Correspondence
}

\section{Propofol, seizure and antidepressants}

To the Editor:

Dr. J.C. Bevan's editorial entitled "Propofol-related convulsions" raised concerns regarding propofol's epileptogenic properties.' Of particular interest was the review of excitatory neurological events following propofol anaesthesia which indicated $46 \%(17 / 37)$ of patients received additional proconvulsant drugs. Here we describe a case of seizure associated with propofol in a patient receiving maprotoline and lithium. Until the mechanisms underlying propofol-induced neuroexcitation are understood, it would seem prudent to avoid using propofol in patients taking drugs that significantly lower the seizure threshold.

A 37-yr-old, 89-kg man was admitted for lumbar microdiscectomy for treatment of a herniated disc. He had a ten-year history of a bipolar mood disorder which was treated with maprotoline $200 \mathrm{mg}$ qhs and lithium 300 $\mathrm{mg}$ od. His lithium therapy was monitored at a peripheral clinic and recent blood concentrations were reported to be within the therapeutic range. Anaesthesia was induced with fentanyl $(100 \mu \mathrm{g})$, d-tubocurarine $(4.5 \mathrm{mg})$, and propofol $(200 \mathrm{mg})$. Shortly after injection of the propofol, the patient complained of a burning sensation in his face. He then became rigid, his back and neck extended and his eyes deviated upward. After $15 \mathrm{sec}$, rhythmic twitching developed in his eyes, arms and hands. The apparent seizure continued for approximately 60 secs until succinylcholine, $120 \mathrm{mg}$, was administered and his trachea was intubated. After several minutes, the patient regained consciousness and the trachea was extubated. Surgery was cancelled and he was transferred to the Postoperative Care Unit for further observation.

An electroencephalogram (EEG) performed two hours later revealed generalized slowing with a 9-10 $\mathrm{Hz}$ alpha rhythm intermixed with theta and low voltage beta rhythm. Some sharp waves, suggestive of a possible irritable focus occurred from the left fronto-temporal region. No spike-slow waves, indicative of epileptiform activity, were observed. An enhanced CT scan of the brain detected no structural abnormalities. The patient was discharged home to await his surgery. An EEG performed two weeks later demonstrated a well-organized alpha rhythm with some intermittent theta activity but none of the focal features that were evident in the previous record.
Maprotoline (Ludiomil) is a tetracyclic antidepressant which initially gained popularity because of its rapid onset, equal efficacy with tricyclics and a lower incidence of side effects. ${ }^{2,3}$ However, subsequent reports revealed that maprotoline was associated with a disproportionately large number of convulsions. ${ }^{4}$ The incidence of seizures was reported to be as high as $15.6 \%$ and was strongly related to dosage. The maximum recommended daily dose for outpatients is $150 \mathrm{mg} \cdot$ day $^{-1}$. Lithium, a drug which alters neuronal second messenger systems and effects the metabolism of biogenic amines, as well as maprotiline, may have predisposed the patient to seizure. ${ }^{5}$ As in most cases, it is not known if the tonic-clonic movements that we observed were a direct effect of propofol or resulted from an interaction between propofol and the antidepressant medication. Should surgery be cancelled and neurological investigation undertaken for patients who develop severe excitatory events following propofol? The question is presented for debate.

The full clinical spectrum of propofol-related neuroexcitatory events will only be clarified with continued reporting. Meanwhile, we suggest that propofol be used with caution in patients taking tetracyclic compounds and other antidepressant medications.

\section{Beverley Orser MD FRCPC}

Don Oxorn MD CM FRCPC

Department of Anaesthesia, Sunnybrook Health Science Centre Toronto, Ontario Canada

\section{REFERENCES}

1 Bevan JC. Propofol-related convulsions (Editorial). Can J Anaesth 1993; 40: 9 805-9.

2 Lipka LJ, Lathers CM. Psychoactive agents, seizure production, and sudden death in epilepsy. J Clin Pharmacol 1987; 27: 169-83.

3 Skowron DM, Stimmel GL. Antidepressants and the risk of seizures. Pharmacotherapy 1992; 12 (1): 18-22.

4 Jabbari B, Bryan GE, Marsh EE, Gunderson CH. Incidence of seizures with tricyclic and tetracyclic antidepressants. Arch Neurol 1985; 42.

5 Schaul N, DeGreef G, Ney GC. Psychotropic drugs and seizures. In: Kane JM, Lieberman JA (Eds.). Adverse Effects of Psychotropic Drugs. New York: The Guilford Press, 1992. 